\title{
Turkish Propolis Extract Increases Apoptosis via Induction of Mitochondrial Membrane Potential Loss in MCF-7 Cells ${ }^{+}$
}

\author{
Sema Misir 1,2,*, Selim Demir ${ }^{3}$, Ibrahim Turan ${ }^{4,5}$ and Yuksel Aliyazicioglu ${ }^{2}$ \\ 1 Department of Biochemistry, Faculty of Pharmacy, Cumhuriyet University, Sivas 58140, Turkey \\ 2 Department of Medical Biochemistry, Faculty of Medicine, Karadeniz Technical University, Trabzon 61080, \\ Turkey; yukselayazici@hotmail.com \\ 3 Department of Nutrition and Dietetics, Faculty of Health Sciences, Karadeniz Technical University, \\ Trabzon 61080, Turkey; selim-demir@hotmail.com \\ 4 Department of Genetic and Bioengineering, Faculty of Engineering and Natural Sciences, Gumushane \\ University, Gumushane 29100, Turkey; ibrahimtrn@gmail.com \\ 5 Medicinal Plants, Traditional Medicine Practice and Research Center, Gumushane University, \\ Gumushane 29100, Turkey \\ * Correspondence: smisir@cumhuriyet.edu.tr; Tel.:+90-346-21910101 (ext. 3913) \\ + Presented at the 2nd International Conference on Natural Products for Cancer Prevention and Therapy, \\ Kayseri, Turkey, 8-11 November 2017.
}

Published: 14 November 2017

\begin{abstract}
Breast cancer is the second most common cancer type among women worldwide. Chemotherapy is the most commonly used strategy against in treatment of breast cancer, but chemotherapeutics also have toxic effects on healthy cells. Natural products have low toxicity, so numbers of chemotherapeutic agents extracted from natural products for treatment of various types of cancers have been increasing for yearly. Propolis is a bee product and contains various polyphenolic compounds. It has been shown that polyphenols affect various signal pathways, such as inhibit cell proliferation, and activate apoptosis, mitochondrial dysfunction. In this study, we aimed to evaluate the effect of Turkish propolis ethanolic extract (EEP) on mitochondrial membrane potential (MMP) alterations, the levels of p53-Ser46 and p53-Ser15 protein in human breast cancer (MCF-7) cells. MMP alterations, protein levels of p53-Ser46 and p53-Ser15 were determined using spectrofluorometric analysis and western blot method, respectively. Results: EEP significantly reduced MMP in dose dependent manner in MCF-7 cells. Moreover, EEP caused increase p53-Ser46 and p53-Ser15 protein levels at $72 \mathrm{~h}$ in these cells. Mitochondrial dysfunction is as an important constituent of apoptosis process. EEP may have apoptotic effects in MCF-7 cells through loss of MMP depend on increased phosphorylation of p53.
\end{abstract}

Keywords: breast cancer; mitochondrial membrane potential; p53; Turkish propolis

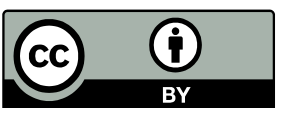

(C) 2017 by the authors. Licensee MDPI, Basel, Switzerland. This article is an open access article distributed under the terms and conditions of the Creative Commons Attribution (CC BY) license (http://creativecommons.org/licenses/by/4.0/). 